\title{
INFLUÊNCIA DA IRRIGAÇÃO INTRAPERITONEAL DE SOLUÇÃO SALINA EM DIFERENTES TEMPERATURAS NA FORMAÇÃO DE ADERÊNCIAS E PREVENÇÃO DE HIPOTERMIA APÓS PNEUMOPERITÔNIO EM RATOS
}

\author{
EFFECT OF INTRAPERITONEAL SALINE SOLUTION IRRIGATIONAT \\ DIFFERENT TEMPERATURES ON ADHESION FORMATION AND \\ HYPOTHERMIA PREVENTION AFTER PNEUMOPERITONEUM IN RATS
}

\author{
Oswaldo Lourenço de Molla Neto ${ }^{1}$ \\ Rodrigo Altenfelder Silva, TCBC-SP ${ }^{2}$ \\ Adhemar Monteiro Pacheco Júnior, TCBC-SP ${ }^{3}$
}

\begin{abstract}
RESUMO: Este estudo experimental em ratos avalia a influência da irrigação da cavidade peritoneal com solução salina isotônica $(0,9 \%)$, em diferentes temperaturas, na formação de aderências peritoneais e prevenção de hipotermia após pneumoperitônio. Foram utilizados 80 ratos divididos em quatro grupos de 20 animais: grupo controle $\left(\mathrm{G}_{1}\right)$ sem irrigação, grupos com irrigação a temperatura ambiente $22,0^{\circ} \mathrm{C}\left(\mathrm{G}_{2}\right)$, a $35,0^{\circ} \mathrm{C}\left(\mathrm{G}_{3}\right)$ e a $45,0^{\circ} \mathrm{C}\left(\mathrm{G}_{4}\right)$. A análise da hipotermia foi realizada através da monitorização da temperatura retal em três diferentes momentos: após a anestesia $\left(\mathrm{T}_{1}\right)$, cinco minutos depois da insuflação de dióxido de carbono $\left(\mathrm{T}_{2}\right)$ e cinco minutos após a irrigação com solução salina $\left(\mathrm{T}_{3}\right)$. Os animais foram sacrificados no $28^{\circ}$ dia de pósoperatório. Observaram-se aderências nos grupos com irrigação, sendo que, com salina à temperatura de $45,0^{\circ} \mathrm{C}$ houve maior formação de aderências $(30,0 \%)$, porém, esta diferença não foi significante. No $\mathrm{G}_{2}$ ocorreu uma queda significante na temperatura média retal quando comparada aos demais grupos, demonstrando que a hipotermia na cirurgia laparoscópica pode ser reduzida com o uso de solução salina aquecida.
\end{abstract}

Unitermos: Aderência, Laparoscopia, Hipotermia

\section{INTRODUÇÃO}

A formação de aderências peritoneais permanece como um importante problema ainda não resolvido uma vez que constitui causa frequente de obstrução intestinal $^{1-3}$. Neste sentido, vários estudos propõem métodos de prevenção ${ }^{4-6}$, utilizando a administração de diferentes substâncias como soluções de cristalóides na cavidade peritoneal ${ }^{7,8}$.

O uso de salina isotônica $(0,9 \%)$ para irrigar a cavidade peritoneal é um procedimento comum em cirurgia, pois, ajuda a remoção de coágulos sanguíneos e tecidos desvitalizados que podem atuar como adjuvantes na gênese de aderências ${ }^{9-11}$. Infusões aquecidas são também recomendadas para prevenir a hipotermia, porém, estudo experimental mostrou que a utilização de líquidos a temperaturas elevadas contribuiria para maior formação de aderências ${ }^{10}$.

A hipotermia nas laparotomias provavelmente resulta da vasodilatação provocada pelos anestésicos, perda de calor para o ambiente, infusão de soluções frias e pela exposição dos órgãos abdominais. Ainda que este último item não faça parte nas videolaparoscopias, a ocorrência de hipotermia é ainda assunto controverso, pois, o dióxido de carbono $\left(\mathrm{CO}_{2}\right)$ utilizado na criação e manutenção do pneumoperitônio poderia desencadeá-la ${ }^{12,13}$. Assim, a irrigação da cavidade peritoneal com substâncias líquidas aquecidas poderia ser um dos meios para sua prevenção.

1. Residente do Departamento de Cirurgia da Faculdade de Ciências Médicas da Santa Casa de São Paulo.

2. Professor Assistente-Doutor do Departamento de Cirurgia da Faculdade de Ciências Médicas da Santa Casa de São Paulo.

3. Professor Adjunto-Doutor do Departamento de Cirurgia da Faculdade de Ciências Médicas da Santa Casa de São Paulo.

Recebido em 5/2/99

Aceito para publicação em 6/12/99

Trabalho realizado no Departamento de Cirurgia da Faculdade de Ciências Médicas da Santa Casa de São Paulo. 
Este estudo experimental foi elaborado com o objetivo de determinar o efeito da solução salina isotônica, com diferentes temperaturas, na formação de aderências peritoneais e na prevenção da hipotermia após pneumoperitônio.

\section{MATERIAL E MÉTODOS}

Este trabalho foi desenvolvido na Unidade de Técnica Cirúrgica e Cirurgia Experimental da Santa Casa de São Paulo. Utilizamos 80 ratos da raça Wistar, machos, pesando entre 250 e $350 \mathrm{~g}$. Observado jejum pré-operatório de 12 horas.

Os animais foram distribuídos em quatro grupos de 20 ratos em cada, sendo:

- Grupo -controle $\left(G_{l}\right)$ - submetido a anestesia e medida da temperatura retal pós-anestésica $\left(\mathrm{T}_{1}\right)$, cinco minutos da anestesia $\left(\mathrm{T}_{2}\right)$ e 10 minutos da anestesia $\left(\mathrm{T}_{3}\right)$;

- Grupo temperatura ambiente $\left(G_{2}\right)$ - anestesia, insuflação de $\mathrm{CO}_{2}$ por cinco minutos seguida pela irrigação da cavidade peritoneal com solução salina isotônica a $22,0^{\circ} \mathrm{C}$ durante cinco minutos;

- Grupo $3\left(G_{3}\right)$ - anestesia, insuflação de $\mathrm{CO}_{2}$ por cinco minutos e irrigação com solução salina isotônica a $35,0^{\circ} \mathrm{C}$ durante cinco minutos;

- Grupo $4\left(G_{4}\right)$ - anestesia, insuflação de $\mathrm{CO}_{2}$ por cinco minutos e irrigação com solução salina isotônica a $45,0^{\circ} \mathrm{C}$ durante cinco minutos. Nos grupos $\mathrm{G}_{2}, \mathrm{G}_{3}, \mathrm{G}_{4}$ mediu-se a temperatura retal pós-anestésica $\left(\mathrm{T}_{1}\right)$, cinco minutos da insuflação de $\mathrm{CO}_{2}\left(\mathrm{~T}_{2}\right)$ e dez minutos da insuflação e cinco minutos da irrigação $\left(\mathrm{T}_{3}\right)$.

$\mathrm{Na}$ anestesia utilizamos a administração intramuscular de Cloridrato de Cetamina e solução aquosa a $2 \%$ de Cloridrato de 2-(2,6-xilino)-5,6-dihidro-4H-1,3-tiazina, e a seguir tricotomia abdominal. Para mensuração da temperatura utilizou-se o sensor do monitor, o qual foi introduzido $2,0 \mathrm{~cm}$ no reto do animal e aferida a temperatura no início do procedimento $\left(\mathrm{T}_{1}\right)$. Procedeu-se pequena incisão na altura da cicatriz umbilical e introdução da agulha de Veress. Realizado o pneumoperitônio com a insuflação de $\mathrm{CO}_{2}$ à temperatura de $21,1^{\circ} \mathrm{C}$ com fluxo de $0,4 \mathrm{~m} / \mathrm{L}$ e pressão de $5 \mathrm{mmHg}$. Após cinco minutos do início do pneumoperitônio foi aferida a temperatura do animal $\left(\mathrm{T}_{2}\right)$.

Passagem do trocarte de 4,0 $\mathrm{mm}$ para colocação da ótica de $30^{\circ}$. Os animais que na inspeção da cavidade apresentaram acidentes na passagem da agulha de Veress e/ou do trocarte foram substituídos. Realizamos então nova punção da cavidade peritoneal sob visão direta, distando $3,0 \mathrm{~cm}$ cranialmente da cicatriz umbilical por onde foram infundidos $40 \mathrm{ml}$ de salina isotônica na cavidade peritoneal, exceto no grupo controle $\left(G_{1}\right)$, onde não se realizou a irrigação. A solução fisiológica na temperatura ambiente se encontrava a $22,0^{\circ} \mathrm{C}\left(\mathrm{G}_{2}\right)$, e foi aquecida e conservada em banho-maria a $35,0^{\circ} \mathrm{C}\left(\mathrm{G}_{3}\right)$ e $45,0^{\circ} \mathrm{C}\left(\mathrm{G}_{4}\right)$. Após cinco minutos da infusão em diferentes temperaturas foi aferida a temperatura retal $\left(\mathrm{T}_{3}\right)$. Síntese da ferida cirúrgica em plano único com náilon 5-0. No pós-operatório imediato os animais foram aquecidos com luz artificial e oferecida água e ração. O sacrifício foi realizado no 28 음a de pós-operatório seguido da necropsia.

As aderências foram macroscopicamente classificadas segundo a presença ou ausência das mesmas. Não foram estabelecidos critérios diferentes para aderências víscero-viscerais, víscero-parietais ou com a cicatriz cirúrgica.

Foram feitas análises de variâncias das diferenças de temperatura $\left(T_{2}-T_{1}\right.$ e $\left.T_{3}-T_{2}\right)$, para verificar se existiam diferenças entre os grupos. Foi utilizado o teste de Tukey considerando-se um valor significante quando $\mathrm{p}<$ 0,05 . Para verificar se existem valores significantes nas proporções de ratos com aderências foi calculado o teste Qui-quadrado com níveis de tolerância de $5 \%$.

\section{RESULTADOS}

Aderências peritoneais foram observadas nos grupos onde realizaram-se a irrigação da cavidade peritoneal (Figura 1). O número de aderências macroscópicas encontradas no $\mathrm{G}_{4}(6 / 20)$ foi superior quando comparado com $\mathrm{G}_{2}(4 / 20)$ e $\mathrm{G}_{3}(4 / 20)$. A distribuição percentual nos diferentes grupos é mostrada no Gráfico 1 .

Foi calculado o teste Qui-quadrado para verificar di-

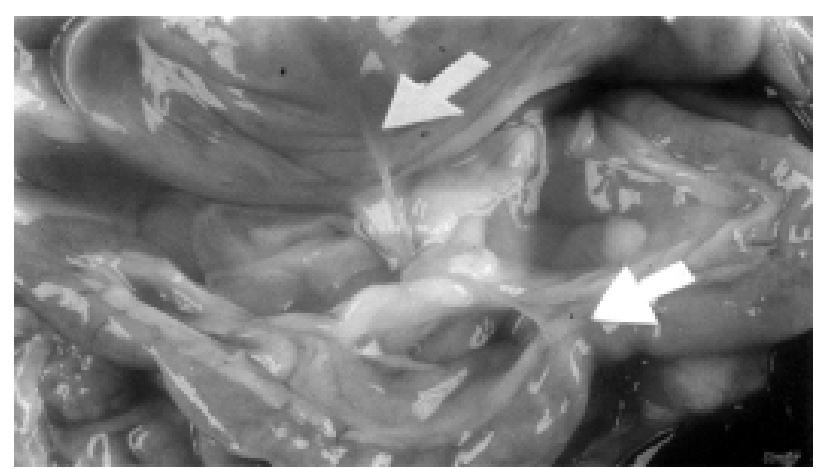

Figura 1 - Detalhes de aderências peritoneais entre alças intestinais.

ferenças das proporções de ratos com aderências entre os grupos, e o resultado não foi significante $\left(x^{2}=6,580\right.$, $\mathrm{p}=0,0865)$. Não ocorreu nenhum óbito durante o período pós-operatório estabelecido neste estudo.

A temperatura média do ambiente nos dias de realização do experimento foi de $22,2^{\circ} \mathrm{C}$. A perda térmica após a insuflação com $\mathrm{CO}_{2}$ ocorreu em todos os grupos que realizaram pneumoperitônio, porém, não houve diferença significante quando comparada com o grupo-controle sem pneumoperitônio.

A análise de variâncias das diferenças de temperaturas entre os grupos mostra que houve modificações significantes apenas entre os momentos $\mathrm{T}_{2}$ e $\mathrm{T}_{3}(\mathrm{p}<0,0001)$. As diferenças $\left(T_{3}-T_{2}\right)$ nos grupos $G_{1}, G_{3}$ e $G_{4}$ não apresentaram alterações significativas entre si. Os achados do $G_{2}$ quando comparado com os demais grupos foi significante, ou seja, quando utilizou-se a irrigação com solução fisiológica a $22,0^{\circ} \mathrm{C}$ a temperatura retal média diminuiu mais do 


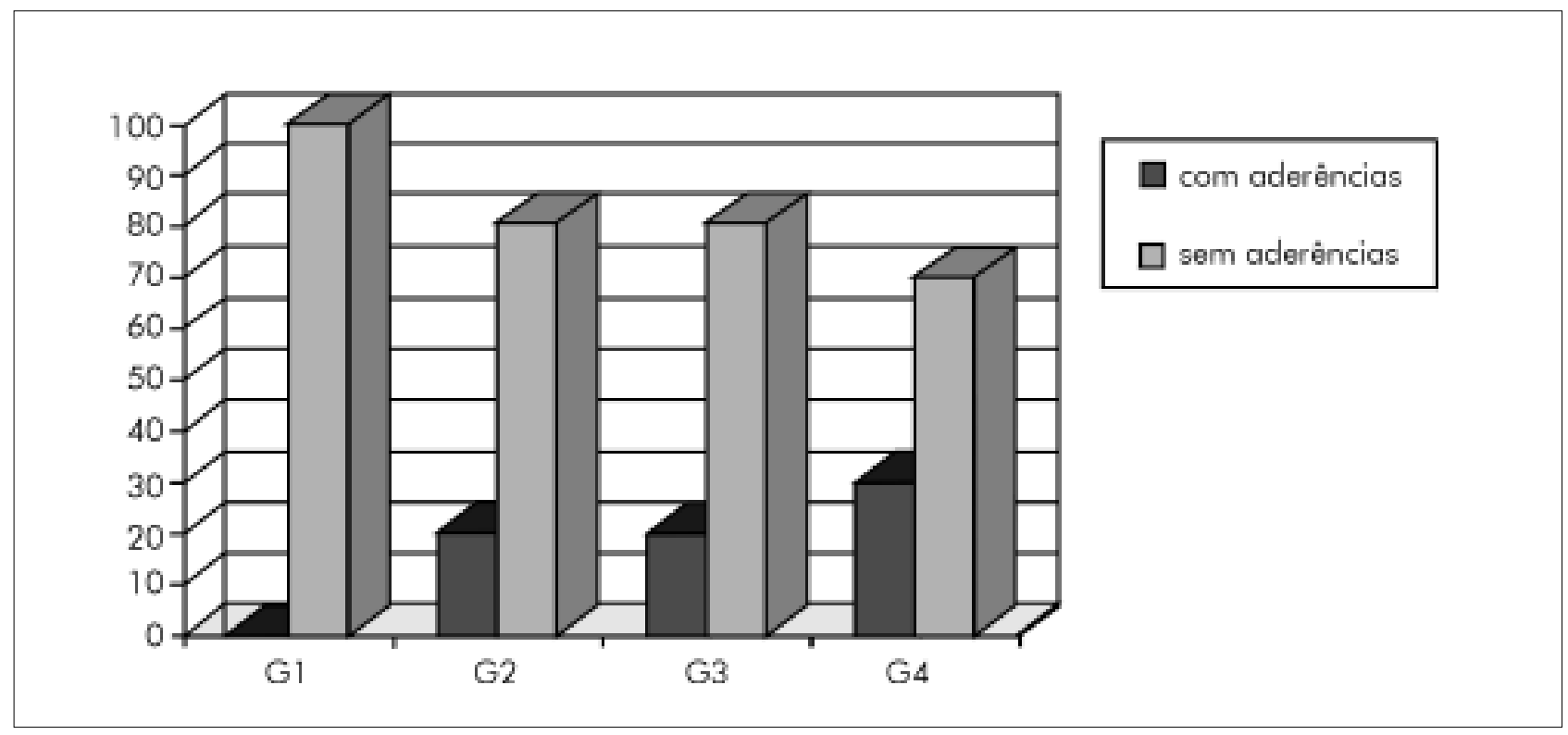

Gráfico 1 - Distribuição percentual dos ratos segundo grupo e aderências.

que nos grupos com solução aquecida (Gráfico 2).

\section{DISCUSSÃo}

A videolaparoscopia é um procedimento cada vez mais utilizado e indicado ${ }^{14-16}$. Várias pesquisas têm procurado avaliar seu efeito na formação de aderências e no desenvolvimento de hipotermia ${ }^{12,17,18}$. Estudos recentes como demonstrado por Luciano et al. ${ }^{19}$ revelam um maior número de aderências observadas na laparotomia que na laparoscopia. Pados et al. ${ }^{18}$ confirmam os efeitos benéficos desta última com relação ao desenvolvimento de aderências. Inúmeras propostas procuram explicar este fato, incluindo menor manipulação de alças intestinais e menor chance de inserção de corpos estranhos na cavidade.

O uso de soluções cristalóides para irrigação intraperitoneal tem sido freqüiente, porém, suas vantagens permanecem controversas ${ }^{10,20-22}$. Grosz et al. ${ }^{23}$ observaram uma redução de aderências com o uso de solução salina. Maingot ${ }^{24}$, entretanto, sugere que a irrigação peritoneal não é justificada, até mesmo na presença de contaminação fecal.

O mecanismo de ação dos cristalóides é através de um efeito mecânico de hidroflutuação separando as superfícies cruentas das vísceras ${ }^{5,9,11}$. O maior inconveniente, entretanto, seria a rápida absorção pelo peritônio, não permitindo esta separação durante o processo de reparação tecidual ${ }^{7}$.

Dentre as diferentes substâncias utilizadas estão a solução salina isotônica e o lactato de Ringer ${ }^{9,25}$. Cohen e Yacobbi ${ }^{11}$, através de experimentos em ratos, ralataram que não existe benefício da irrigação com lactato de Ringer na prevenção de aderências. O presente estudo escolheu a solução salina isotônica pelo fato de ser amplamente utilizada no nosso meio, por ter baixo custo e pela

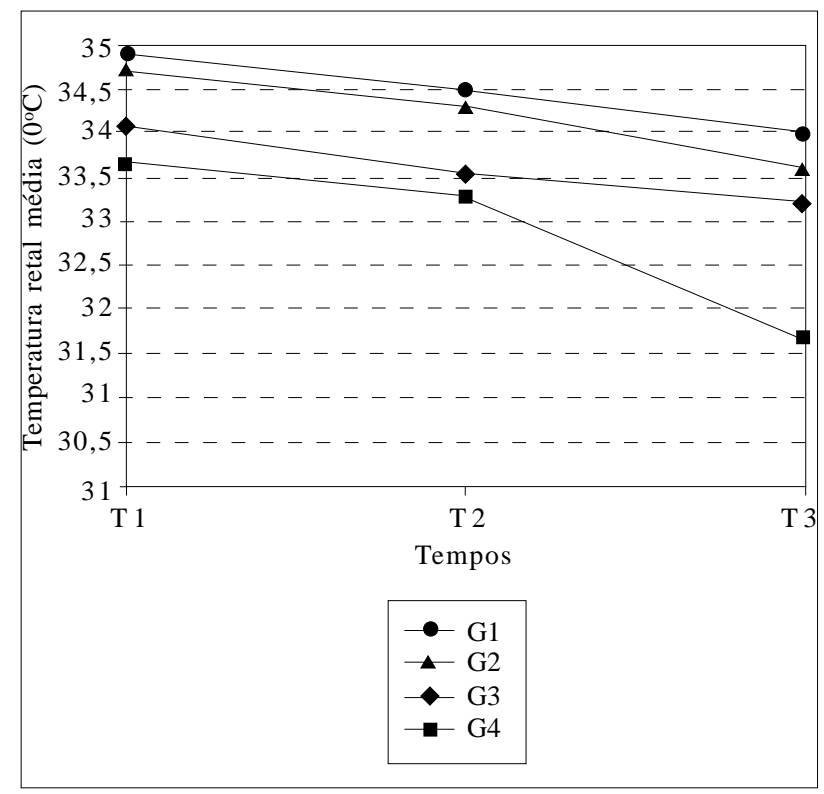

Gráfico 2 - Médias da temperatura retal por grupo e momento.

facilidade de obtenção da mesma.

Uma das dificuldades em se conduzir uma pesquisa sobre a formação de aderências têm sido a falta de uniformidade nas classificações e interpretação das aderências encontradas na literatura ${ }^{6,10,25,26,27}$. Neste estudo a necropsia foi realizada por dois observadores e os resultados foram avaliados de maneira simples fundamentados nos seguintes achados: ou o animal apresentava aderências ou não, independentemente do tamanho e das estruturas envolvidas ${ }^{23}$.

Estabelecemos aleatoriamente o período de pós- 
operatório em 28 dias para sacrificar os animais uma vez que existem estudos sobre a patogênese das aderências, que demonstram que após um período de três a quatro dias da agressão peritoneal o exsudato fibrinoso liberado pelas células mesoteliais do peritônio é reabsorvido. Caso não ocorra este processo, haverá a organização da fibrina e formação de aderências permanentes. Por volta de três semanas as aderências estão histologicamente estabilizadas e começa o processo de fibrose com evolução persistente ${ }^{4,7,9,18,28,29}$.

A temperatura e o volume da solução que deve ser utilizada é muito variável, acreditando-se que a aquecida sempre deve ser preferida, a fim de se evitar a hipotermia peroperatória. Segundo Kappas et al. ${ }^{10}$, em estudo experimental, mostraram que a temperatura da solução quando acima de $37,0^{\circ} \mathrm{C}$ propicia uma maior formação de aderências.

Optamos pela comparação de três temperaturas diferentes, $22,0^{\circ} \mathrm{C}$ que corresponde a ambiente, $35,0^{\circ} \mathrm{C}$ que é considerada normal para o animal e $45,0^{\circ} \mathrm{C}$ que seria considerada uma temperatura elevada. Os resultados obtidos demonstraram não haver diferenças significantes com relação à formação de aderências entre os grupos, embora, a porcentagem no grupo a $45,0^{\circ} \mathrm{C}$ $(30,0 \%)$ tenha sido superior que nos demais.

A hipotermia no peroperatório é decorrente dos efeitos da anestesia que parecem modificar a temperatura corpórea pela alteração intrínseca dos mecanismos termorreguladores do hipotálamo associados a uma série de outros fatores como a temperatura da sala cirúrgica, a ventilação prolongada com gases secos, a infusão de líquidos frios e a exposição da cavidade abdominal ${ }^{12,14-16}$.

Na cirurgia videolaparoscópica, a não exposição dos órgãos poderia fazer com que o grau de hipotermia peroperatório fosse menor. No entanto, a realização e manutenção do pneumoperitônio com gases a baixas temperaturas, particularmente o $\mathrm{CO}_{2}$, permitiria uma queda térmica corpórea maior. Ott ${ }^{15} \mathrm{e}$ Bessell ${ }^{14}$ mostraram que a insuflação de $\mathrm{CO}_{2}$ causa queda significante na temperatura corporal e que a utilização de gás aquecido protege contra a hipotermia. Por outro lado, Huntington e Le Master ${ }^{30}$ relatam que a perda térmica provocada pelo $\mathrm{CO}_{2}$ na laparoscopia é insignificante. Moore et al. ${ }^{17}$ estudaram a incidência e etiologia da hipotermia durante a laparoscopia e o valor da irrigação da cavidade peritoneal com solução de cristalóide aquecida e mostraram que a hipotermia é decorrente de múltiplos fatores e que o uso de solução aquecida parece ser efetiva no combate a mesma. Os resultados obtidos no presente estudo indicam que a solução salina aquecida ajuda a prevenir a hipotermia na cirurgia laparoscópica.

Em função das graves repercussões da hipotermia no paciente cirúrgico, como as arritmias cardíacas, este método de prevenção deve ser lembrado, contribuindo para a redução das taxas de morbimortalidade dos procedimentos videolaparoscópicos.

Os resultados obtidos nas condições de execução desta pesquisa permitem concluir que a irrigação da cavidade peritoneal com solução salina isotônica aquecida até $45,0^{\circ} \mathrm{C}$ não promove a formação de um número significante maior de aderências peritoneais e que a hipotermia provocada pela anestesia e pelo pneumoperitônio pode ser reduzida com o uso desta solução na irrigação da cavidade peritoneal.

\section{REFERÊNCIAS}

\begin{abstract}
Adhesions following abdominal surgery have plagued surgeons for years. Intra-operative irrigation of the peritoneal cavity with isotonic saline solution is a common procedure in gastrointestinal surgery. Although warm saline is often recommended to prevent hypothermia, accurate temperature control is rarely observed. Recent experimental studies are correlating saline temperature with adhesion formation and it has been shown that when saline temperature exceeds $37,0^{\circ} \mathrm{C}$ it increases the possibilities of adhesions. Hypothermia is frequent during laparoscopy due to the insufflation of cold carbon dioxide to induce pneumoperitoneum. Most laparoscopic procedures also use irrigation with physiologic solutions, such as normal saline. Our purpose was to evaluate the influence of intraperitoneal isotonic saline solution irrigation, at different temperatures, on adhesion formation and hypothermia prevention after pneumoperitoneum. Eighty rats were divided in four groups of 20 animals each: control group $\left(G_{1}\right)$ with no irrigation, $G 2$ group with irrigation at ambient temperature $22,0^{\circ} \mathrm{C}, \mathrm{G} 2$ at $35,0^{\circ} \mathrm{C}$ and $\mathrm{G} 4$ at $45,0^{\circ} \mathrm{C}$. Temperature analysis was accomplished through a rectal probe in three different moments: after anesthesia $\left(T_{1}\right)$, five minutes after carbon dioxide insuflation $\left(T_{2}\right)$ and five minutes after saline irrigation $\left(T_{3}\right)$. The animals were sacrificed in the $28^{\text {th }}$ day of postoperative. Adhesions were observed in all groups with irrigation and a greate adherence formation $(30 \%)$ was observed at $45,0^{\circ} \mathrm{C}$. Even so, this difference was not significant. In the $G_{2}$ a significant fall occurred in the rectum average temperature when compared with the other groups demonstrating that the hypothermia can be avoided with the use of warmed saline solution in laparoscopic surgery.
\end{abstract}

Key Words: Adhesion, Laparoscopy, Hypothermia. 
1. Ellis $\mathrm{H}-$ The causes and prevention of intestinal adhesions. Br J Surg 1982; 69: 241-243.

2. Menzies D, Ellis H - Intestinal obstruction from adhesions How big is the problem? Ann R Coll Surg Engl 1990; 72: 60-63.

3. Weibel MA, Majno G - Peritoneal adhesions and their relation to abdominal surgery. A post mortem study. Am J Surg 1973; 126: 345-353.

4. Buckman RF, Woods M, Sargent L, et al. - A unifying pathogenetic mechanism in the etiology of intraperitoneal adhesions. J Surg Res 1976; 20:1-5.

5. diZerega GS - The cause and prevention of postsurgical adhesions: a contemporary update. Prog Clin Biol Res 1993; 381: 1-18.

6. Ryan GB, Grobéty J, Majno G - Postoperative peritoneal adhesions. A study of the mechanisms. Am J Pathol 1971; 65: 117-138.

7. Aldrighi JM, Wehba S, Donadio N, et al. - Prevenção de aderências pélvicas. Reprodução 1987; 2: 140-143.

8. Pereira-Lima L, Kalil NA, Rhoden EL, et al. - Efeito de drogas antiinflamatórias e antifibrosantes sobre aderências intra abdominais pós-operatórias, em um modelo experimental. Acta Cir.Bras. 1992; 7(1): 31-34.

9. diZerega GS, Campeau D - Use of instillates to prevent intraperitoneal adhesions. Infertil Reprod Med Clin North Am 1994; 5(3): 463-478.

10. Kappas AM, Fatouros M, Papadimitriou K et al. - Effect of intraperitoneal saline irrigation at different temperatures on adhesion formation. Br J Surg 1988; 75: 854856.

11. Yaacobi Y, Goldberg E - Effect of Ringer's lactate irrigation on the formation of postoperative abdominal adhesions. J Invest Surg 1991; 4: 31-36.

12. Castillo V, Gutiérrez-Crespo A, Suárez F, et al - Variaciones de la temperatura corporal en el curso de colecistectomías laparoscópicas. Rev. Esp. Anestesiol. Reanim. 1996; 43: 201-203.

13. Ott DE - Correction of laparoscopic insufflation hypothermia. J Laparoendosc Surg 1991; 1(4): 183-186.

14. Bessell JR, Karatassa A, Patterson JR et al. - Hypothermia induced by laparoscopic insuflation. Surg. Endosc 1995; 9: 791-796.

15. Ott DE - Laparoscopic hypothermia. J Laparoendosc Surg 1991; 1(3): 127-131

16. Seitzinger MR, Dudgeon LS - Decreasing the degree of hypothermia during prolonged laparoscopic procedures. J Reprod Med 1993; 38(7): 511-513.

18. Pados G, Devroey P - Adhesions. Curr Opin Obstet Gynecol
1992; 4: 412-418.

19. Luciano A, Maier D, Koch E et al. - A comparative study of postoperative adhesions following laser surgery by laparoscopy versus laparotomy in rabbit model. Obstet Gynecol 1989; 74: 220-224.

20. Ambrose NS, Donovan IA, Derges S et al - The efficacy of peritoneal lavage at elective abdominal operations. $\mathrm{Br}$ J Surg 1982; 69: 143-144.

21. Nyström P, Johansson L, Lennquist S - Intra-operative irrigation of the peritoneal cavity in experimental post-traumatic peritonitis. Acta Chir Scand 1983; 149: 509-515.

22. Sortini A, Fabbri N, Balboni PG et al. - Studio sperimentale sul lavaggio postoperatorio: standardizzazione del metodo. Minerva Chir 1996; 51: 443-446.

23. Grosz C, Aka E, Zimmer J et al - The effect of intraperitoneal fluids on the prevention of experimental adhesions. Surgery 1996; 60: 1232-1234.

24. Maingot R - "Acute peritonitis". In: Maingot R - Abdominal Operations, 6 th edn. New York: Appleton-CenturyCrofts, 1974, pp 1416-1420.

25. Koh IHJ, Guimarães Neto JF, Bijotti JR et al - Aderências peritoneais produzidas experimentalmente em ratos. Acta Cir. Bras 1988; 3(3): 73-79.

26. La Morte AI, Diamond MP - Adhesion formation after laparoscopy. Prog Clin Biol Res 1993; 381: 51-58.

27. Phillips RKS, Dudley HAF - The effect of tetracyline lavage and trauma on visceral and peritoneal ultrastructure and adhesion formation. Br J Surg 1984; 71: 537-539.

28. Kappas AM, Papadimitriou CS, Fatouros M et al - Histologic changes in adhesiogenesis following intraperitoneal saline irrrigation at different temperatures. Res Surg 1990; 2(3): 148-151.

29. Cohen BM, Heyman T, Mast D - Use of intraperitoneal solutions for prevent pelvic adhesions in rats. J Reprod Med 1983; 28: 649-653.

30. Huntington TR, Le Master CB - Laparoscopic hypothermia: heat loss from insufflation gas flow. Surg Laparosc Endosc 1997; 7(2): 153-155.

\section{ENDEREÇO PARA CORRESPONDÊNCIA}

Dr. Oswaldo Lourenço Molla Neto

Rua Dr. Cesário Motta Jr., 61

01221-020 - São Paulo-SP 